\title{
Living on the edge: Rossby wave instability and HFQPOs in black hole binaries
}

\author{
P. Varniere ${ }^{1,2}$, F. H. Vincent ${ }^{3}$, and F. Casse ${ }^{1,2}$ \\ 1 Université de Paris, CNRS, Astroparticule et Cosmologie, 75013 Paris, France \\ e-mail: varniere@apc.univ-paris7.fr \\ 2 AIM, CEA, CNRS, Université Paris-Saclay, Université Paris Diderot, Sorbonne Paris Cité, 91191 Gif-sur-Yvette, France \\ 3 Observatoire de Paris/LESIA, 5 Place Jules Janssen, 92195 Meudon Cedex, France
}

Received 25 February 2020 / Accepted 14 April 2020

\begin{abstract}
Context. The Rossby wave instability (RWI) has been proposed to explain the origin of the high-frequency quasi-periodic oscillations observed in the X-ray emission of astrophysical systems harbouring black holes. Recent numerical computations have proven that the RWI does exist in a general relativistic context and that its presence is associated with a time-variable X-ray emission from the disc. Aims. Using our new Numerical Observatory of Violent Accreting system, NOVAs, we explore the way the RWI impacts an accretion disc orbiting a spinning black hole under realistic astrophysical conditions. Our aim is to study the impact of the presence of the RWI in the very inner part of the accretion disc on known observables and explore some possibly new ones.

Methods. We present the first full general relativistic hydrodynamical simulations of the RWI occurring at the last stable orbit of an accretion disc orbiting around a Kerr black-hole. Those simulations, coupled with a full general relativistic ray-tracing, have allowed us to directly compare our simulations with the observables we obtained from the X-ray emission of the disc.

Results. Our study shows, for the first time, that the RWI naturally arises near the inner edge of an accretion disc whenever it gets close to its last stable orbit, as predicted analytically. From there, we show that not only does the RWI create a visible timing feature but it also impacts the energy spectrum of the source, which exhibits a high energy extension due to the presence of hot vortices generated by the RWI in the disc. Our study also shows that systems with the RWI present at the inner edge of the disc only exhibit similar behavior to systems in which HFQPOs have been detected.
\end{abstract}

Key words. X-rays: binaries - accretion, accretion disks

\section{Introduction}

While black-hole binaries often display extreme variability, their most sought-after behavior is the rather elusive high-frequency quasi-periodic oscillation (HFQPO). Those narrow peaks have been detected in the X-ray power-density spectra (PDS) of eight different black-hole binaries (GRO J1655-40, GRS 1915+105, XTEJ1550-564， H1743-322， 4U 1630-47， XTE J1650500, XTEJ1859+226 IGRJ17091-3624, e.g. Remillard \& McClintock 2006; Altamirano \& Belloni 2012; Belloni et al. 2012, and references therein), ranging from as low as $27 \mathrm{~Hz}$ in GRS 1915+105 (Belloni et al. 2001) up to a few hundred $\mathrm{Hz}$ (a maximum of $450 \mathrm{~Hz}$ was seen in GRO J1655-40; Remillard $\&$ McClintock 2006). One of the reason HFQPOs garner so much interest is that their frequencies typically lie in the frequency range associated with the Keplerian motion of the last stable orbit around the central black-hole. Hence, a better understanding of their origin may give us access to what is happening in the innermost region of the accretion disc and, in particular, to the effect of strong gravity ${ }^{1}$.

Nevertheless, even if their existence has stimulated a lot of interest on the theoretical side, no model has yet gained wide acceptance, in part because of the lack of a direct comparison between models and observations. On one hand, those HFQPOs are relatively weak and the rarest of the observed QPOs, which

\footnotetext{
1 Relativistic effects, and their influences on the properties of low frequency QPOs, are, for example, discussed within the context of the accretion-ejection instability in Varniere et al. (2012).
}

makes them hard to detect. On the other, most of the theoretical models have not yet focused on direct comparisons, preferring instead to start by comparing one or more prominent aspects of the observed data. In this paper, we will rectify that last part by studying the "observed" characteristics of one of the proposed models for HFQPOs -that is, the Rossby-Wave Instability (RWI). Across a series of recent papers (Casse et al. 2017; Casse \& Varniere 2018), we have studied how general relativity (GR) affects disc instabilities, such as the RWI, and found that except for high spin when the instability occurs inside $4 r_{g}$, its behavior is similar to and well-approximated by its Newtonian equivalent. Furthermore, in Varniere et al. (2019) we presented our Numerical Observatory of Violent Accreting systems (NOVAs) which is the perfect tool for performing the necessary direct comparison between the RWI "observable" and the X-ray data.

In Sect. 2 of this paper, we present the numerical tools that compose NOVAs. In Sect. 3, we show for the first time that the RWI is indeed naturally triggered when the accretion disc reaches its last stable orbit, as was predicted in Tagger \& Varniere (2006). Section 4 focuses on the imprint of the RWI on the X-ray emission, from timing analysis to the energy spectrum, in search of potential indirect detection of HFQPOs.

\section{RWI simulations: from the source to the observer}

NOVAs is composed of a fluid numerical code, GRAMRVAC, which provides general relativistic hydrodynamical simulations (GRHD) of the system and a general relativistic ray-tracing code, 
gyoto, that computes the emission of the simulated disc measured by a distant observer. This enables us to follow the evolution of the RWI as it is triggered in an accretion disc orbiting around a black hole in the frame of a distant observer.

\subsection{The necessity for a full general relativistic framework}

The RWI develops wherever an extremum of the vortensity occurs (defined by $\mathcal{L}=(\nabla \times V)_{\perp} / \rho$ with $\perp$ denoting the component in the direction perpendicular to the disc, $V$ the velocity and $\rho$ the density). While this criterion is rarely met in accretion discs, a natural place for this to occur is near the last stable orbit around a black hole where the epicyclic frequency of the flow (proportional to its vortensity) experiences an extremum.

Such an occurrence was first predicted by Tagger \& Varniere (2006) and then explored further in Vincent et al. (2013) but, in both cases, it was done using a pseudo-Newtonian approach for the purpose of mimicking the general relativistic effects upon the rotational velocity only. If we want to fully explore the behavior of the RWI around black holes, and especially its ability to explain fast variability (Varniere et al. 2019), we therefore need to take into account all general relativistic effects upon both the fluid and its radiative emission.

\subsection{GRAMRVAC and general relativistic hydrodynamical simulations}

The GRAMRVAC code solves general relativistic hydrodynamics equations, including conservation of mass and momentum. Such equations are expressed while taking into account spacetime geometry around a rotating, uncharged black hole thanks to the Kerr metric (Kerr 1963). Therefore, the general expression of any line element $d s$ in a $(3+1)$ splitting in Boyer-Lindquist coordinates is:

$\mathrm{d} s^{2}=-\alpha^{2}(c \mathrm{~d} t)^{2}+\gamma_{i j}\left(\mathrm{~d} x^{i}+\beta^{i} c \mathrm{~d} t\right)\left(\mathrm{d} x^{j}+\beta^{j} c \mathrm{~d} t\right)$,

where $\alpha$ is the lapse function, $\beta^{i}$ is the shift vector and $\gamma_{i j}$ is the spatial metric tensor (see e.g. Casse \& Varniere 2018, for a definition of these quantities that involve the dimensionless spin parameter $-1 \leq a=J c / G M \leq 1$, where $J$ and $M$ are the angular momentum and mass of the black hole, respectively). In our notation, Greek letters stand for indices ranging over all coordinates while Latin letters are restricted to spatial coordinates only. We denote $\gamma$ as the determinant of the spatial metric tensor.

Defining $\rho$ as the proper density of the gas and $v^{i}$ its contravariant Eulerian velocity components (normalised to the speed of light $c$ ), we can express the aforementioned conservation laws as:

$$
\begin{aligned}
\partial_{t}(\sqrt{\gamma} D)+\partial_{j}( & \left.\sqrt{\gamma} D\left(\alpha v^{j}-\beta^{j}\right)\right)=0 \\
\partial_{t}\left(\sqrt{\gamma} S_{i}\right)+\partial_{j}( & \left.\sqrt{\gamma}\left[S_{i}\left(\alpha v^{j}-\beta^{j}\right)+\alpha P \delta_{i}^{j}\right]\right) \\
= & \sqrt{\gamma}\left\{-\left(W^{2} \rho h c^{2}-P\right) \partial_{i} \alpha\right. \\
& \left.+\frac{\alpha}{2}\left(S^{j} v^{k}+P \gamma^{j k}\right) \partial_{i} \gamma_{j k}+S_{j} \partial_{i} \beta^{j}\right\},
\end{aligned}
$$

where $W=\left(1-v_{i} v^{i}\right)^{-1 / 2}$ is the Lorentz factor of the gas, $D=W \rho$ is the relativistic mass density, $W^{2} \rho h c^{2}$ is the relativistic enthalpy of the fluid, and $P$ is the thermal pressure. The momentum of the fluid is defined as $S_{i}=W^{2} \rho h c^{2} v_{i}$. Taking into account the full energy conservation requires including heating and cooling mechanisms which would involve physics beyond hydrodynamics such as high-energy kinetic theory and radiative transfer.
One way to bypass this complicated machinery is to use a simplified prescription such as the one proposed by Mignone \& McKinney (2007) in their Eq. (13), which mimics the exact solution of relativistic gas thermodynamics. The prescription relating the thermal pressure to the density, $P=C_{o} \rho^{\tilde{\gamma}}$ where $C_{o}$ and $\tilde{\gamma}$ are two positive constants, is here used to replace the energy conservation equation only. In the end if one considers the energy equation, it will still be required to provide the link between the internal energy of the gas and the thermal pressure as in Mignone \& McKinney (2007), for example.

Similarly to what was done in Casse et al. (2017), Casse \& Varniere (2018), in this paper, we consider vertically integrated accretion disc configurations. Since accretion discs in $\mathrm{X}$-ray binaries are likely to be thin, this approach provides reliable results compared to full 3D simulations. In Casse et al. (2017), we validated this framework by showing that 2D and 3D simulations of the RWI around non-spinning black holes lead to very similar results, as expected, because the vertical gravitational force near the equatorial plane weakly depends on the $\theta$ coordinate over the whole disc height.

The initial conditions defining our simulations consider a surface density $\sigma(r)$ covering the entire simulation box are expressed as:

$\sigma(r)=\sigma_{o}\left(\frac{r_{o}}{r}\right)^{3 / 4}$

The radial equilibrium of the disc is obtained through the balance of the centrifugal force, the gravity of the black hole, as well as the thermal pressure gradient of the gas. The rotational velocity is then expressed as:

$$
v^{\varphi}=\frac{-\partial_{r} \beta^{\varphi}+\sqrt{\left(\partial_{r} \beta^{\varphi}\right)^{2}+2 \alpha \partial_{r} \gamma_{\varphi \varphi}\left(\partial_{r} \alpha+\alpha \frac{\partial_{r} P}{\sigma h W^{2}}\right)}}{\alpha \gamma^{\varphi \varphi} \partial_{r} \gamma_{\varphi \varphi}} .
$$

In our simulations, we use the same radial pressure profile $P$ as in our previous study, namely the aforementioned power-law prescription, with the parameters $C_{o}=1.8 \times 10^{-4}$ and $\tilde{\gamma}=3$. Such values are consistent with a thin disc whose aspect ratio is $H / r \sim 4 \times 10^{-2}$. It is noteworthy that in our framework, the disc scale height $H$ is evaluated using the assumption that the vertical pressure gradient balances the vertical gravity.

The grid resolution is $980 \times 600$ cells for each simulation while the spatial domain ranges from $\frac{2}{3} r_{\text {LSO }}$ to $4 r_{\text {LSO }}$ and $\phi \epsilon$ $[0,2 \pi]$ where $r_{\text {LSO }}$ is the radius of the last stable circular orbit which varies depending on black hole spin. The radial boundaries are continuous, meaning the value of physical quantities is simply copied into the boundary cells (i.e. with a zero gradient), while azimuthal boundaries are periodic. We used a Harten, Lax, and van Leer (HLL) solver linked to a Koren slope limiter. A typical simulation requires approximately several tens of thousands of time steps and lasts a few hundreds of CPU-hours.

\subsection{General relativistic ray tracing by gyoto}

The ray-tracing part of NOVAs is performed using the opensource $^{2}$ gyoto code. It uses a Runge-Kutta-Fehlberg adaptivestep integrator at order $7 / 8$ (meaning that the method is 8 th order, with an error estimation at the 7 th order) to integrate the geodesic equation and trace photons backwards in time. We can compute the light curve (total flux as function of time) by adding all the pixels weighted by their respective solid angle, or the energy spectrum, by keeping the energy bin.

2 Freely available at http://gyoto.obspm.fr 

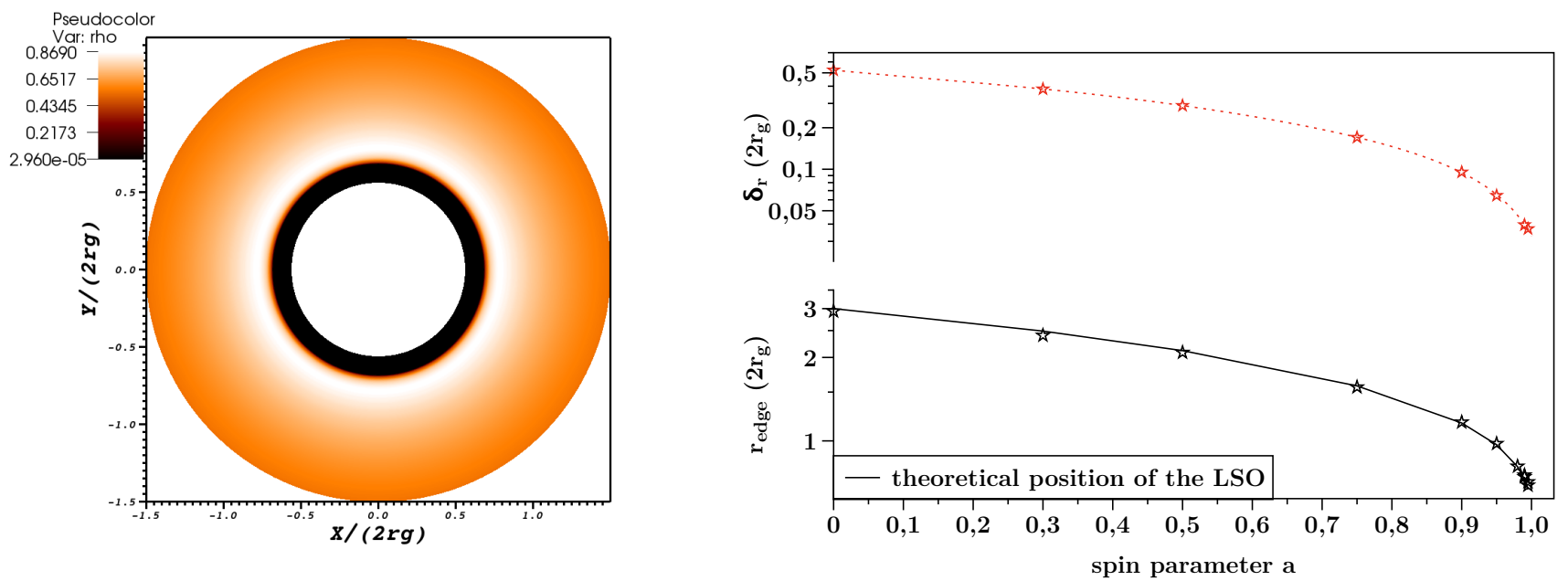

Fig. 1. Left: surface density snapshot from the GRAMVAC simulation of a disc orbiting a $a=0.995$ black hole. At this later stage the initial uniform disc has turned into a disc exhibiting an inner edge whose location coincides with the last stable orbit around the black hole. Right: evolution of the density fit parameters as a function of the spin of the black hole, namely $\delta_{r}$ as the thickness of the edge and $r_{\text {edge }}$ as its position as defined in Eq. (5).

A backwards-integrated photon is traced until the accretion disc is reached with the local temperature derived from GRHD simulation by considering the relation $T(\rho)=T_{\text {inner }}\left(\sigma / \sigma_{\text {inner }}\right)^{\gamma_{o}}$ where $\sigma$ is the local density interpolated from the grid, $\sigma_{\text {inner }}$ is the density at the inner radius of the disc, $T_{\text {inner }}$ is the temperature at the inner radius, fixed at $T_{\text {inner }}=10^{7} \mathrm{~K}$, and $\gamma_{o}$ is the adiabatic index, fixed at $\gamma_{o}=5 / 3$. The GRHD simulation outputs allow us to compute, through the use of the Planck function evaluated for the local temperature, the emission in the local frame of the gas which we use to compute the corresponding intensity measured by a distant, static observer.

\section{RWI growth and evolution near the last stable orbit around a black hole}

In this section, we present our approach to study the occurrence of the RWI when the inner edge of an accretion disc orbiting around a black hole reaches its last stable orbit. This approach consists of two steps, the first one being the formation of the inner edge of the disc while the second one is related to the growth of the RWI.

\subsection{Formation of an inner edge at the last stable orbit of an accretion discs around spinning black holes}

While numerous simulations of the RWI have been previously done either in the context of Newtonian (Lovelace \& Romanova 2014) or Kerr metrics (Casse et al. 2017; Casse \& Varniere 2018), the process for reaching physical conditions that are consistent with an extremum of vortensity in full GR is yet to be explored. Indeed, the first step to running self-consistent simulations of the RWI is to to check for the ability of an extremum of vortensity to occur near the last stable orbit of black-hole as was theorised before (Tagger \& Varniere 2006).

We first use GRAMVAC to run simulations to form, self consistently, the inner edge of the disc around a Kerr black hole. For that purpose, we use the initial conditions where the simulation box is full of gas orbiting the black-hole and we let it evolve until we obtain a stationary flow. We set the physical quantities as mentioned above so that the matter in the disc is very close to equilibrium. However, tiny numerical errors in the initial state act as perturbations that lead to unstable trajectories for the gas located below the last stable orbit. As a result, all matter in the central area is rapidly falling into the black hole, which leads to the formation of an inner edge of the disc. An example of the outcome of such simulations is displayed on Fig. 1 where, in the left panel, we see a late-time snapshot of a disc formed around a black hole whose spin parameter is $a=0.995$. In our simulations, we observe the formation of a sharp inner edge of the disc density close to the theoretical last stable orbit below which all matter has been rapidly accreted.

In order to quantify the position and sharpness of the disc inner edge, we fit the surface density with function $\sigma_{\text {fit }}(r)$,

$\sigma_{\text {fit }}(r)=\frac{\sigma_{o}}{r^{3 / 4}}\left(1+\tanh \left(\frac{r-r_{\text {edge }}}{\delta_{r}}\right)\right)$.

The right panel of Fig. 1 shows the evolution of the disc edge location and sharpness as a function of the spin for our simulated discs. We see that the inner edge location is indeed very close to the theoretical radius of the last stable orbit and that, as the spin increases, the inner edge of the disc gets sharper and thinner. It is noteworthy that for the last two points of the plot in the right panel (corresponding to spins of 0.99 and 0.995), were done with a different setup. Indeed, our chosen resolution does not allow us to fully resolve the sharpness of the inner edge. In order to palliate that limitation we chose to study those two cases with a smaller disc rather than dramatically increasing the spatial resolution and hence the computation time required. As a result, those simulations will not appear in the comparison plots related to the instability as they have a different setup. However they still contribute to proving the ability of an accretion disc to trigger the RWI at the last stable orbit around a fast spinning Kerr black hole.

\subsection{Living on the Edge: the RWI near the last stable orbit}

The purpose of our simulations is to study the growth and evolution of the RWI that is triggered close to the inner edge of an accretion disc located at the last stable orbit around any spinning black hole. We have presented a way of obtaining such a configuration in the previous section for a series of increasing spins so that now we can explore how the RWI develops by adding small 

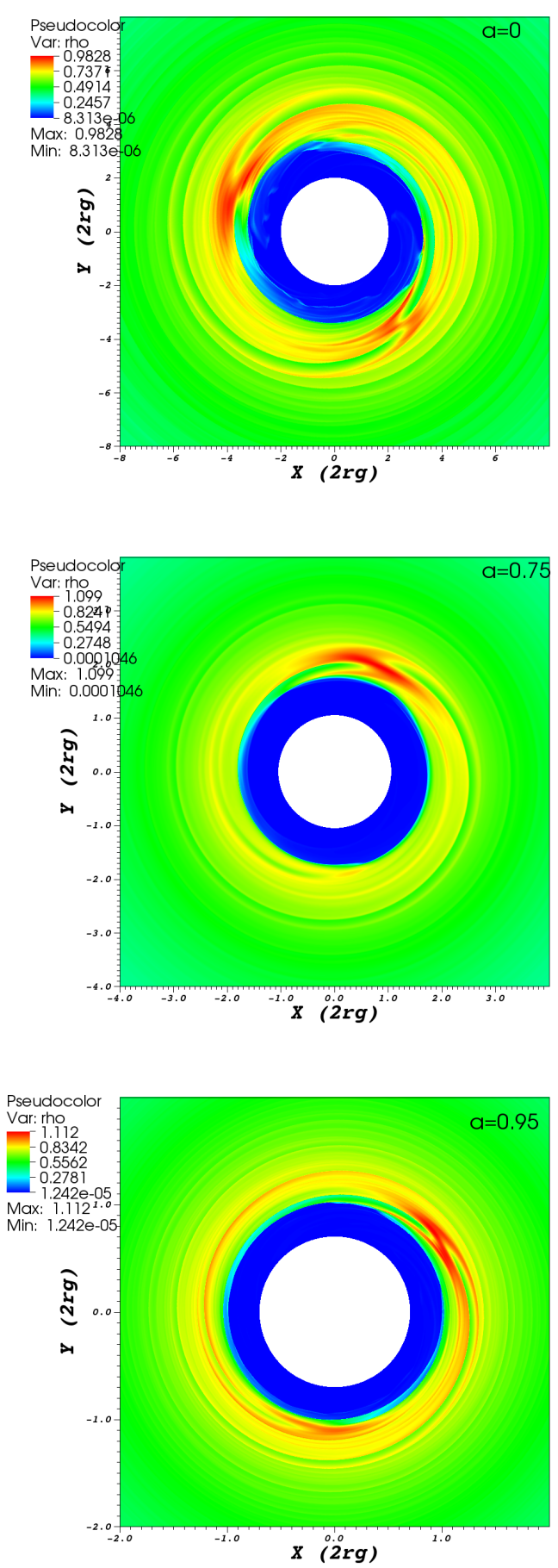

Fig. 2. Snapshots of the RWI developing at the inner edge of a disc orbiting a black hole of spin $a=\{0,0.75,0.95\}$. Those snapshots emphasise a few of the typical behaviors seen for all spins, with one or more vortices that merge with time.

random velocity perturbations $\left(\sim 10^{-5}\right.$ of the rotational velocity) around the maximum of vortensity, namely close to the last stable orbit.

In order to assess any spin impact, we ran a set of similar simulations with increasing spin parameters $a=$ $\{0,0.3,0.5,0.75,0.95,0.98,0.99\}$ while zooming in on the location of the last stable orbit where the RWI is expected. In Fig. 2, we show a few selected cases from our simulation to showcase how similarly the RWI develops for all the spins but also how different modes are visible during the evolution of the

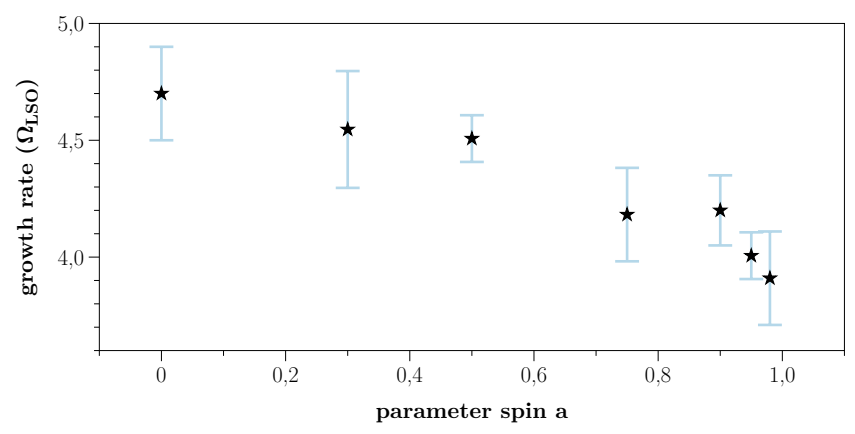

Fig. 3. Evolution of the growth rate of the RWI developing at the inner edge of a disc orbiting at the last stable orbit of a black hole as function of spin.

instability. Which mode appears and how it evolves with time was thoroughly studied in Casse et al. (2017), with their Fig. 9, showing the growth rate of the different modes and how most of them merge toward lower modes by the end of the linear phase. The timescale on which this happens was presented as function of spin in Fig. 9 of Casse \& Varniere (2018), demonstrating that, based on our current observational capability, we cannot differentiate between spins for low-mass black-hole binaries.

In every simulation, the RWI develops close to the last stable orbit, the exact position depending on the shape of the density profile at the inner edge of the disc, hence, on the spin of the black hole (see Fig. 1). One noticeable thing in the few examples in Fig. 2 is that as the spin increases, the RWI features become narrower and for a spin above $a=0.99$ we cannot resolve it in a reasonable computing time. This is in agreement with the results of Fig. 1 which shows that the inner edge is more peaked and narrow as the spin increases.

The next step is to obtain the growth rate as a function of spin. In order to do so, we follow the evolution of the maximum relative density fluctuations amplitude, $\Delta \sigma / \sigma$, as function of time for each spin case and measure the slope in the linear evolution of the instability. This is shown in Fig. 3 for all of our simulations with similar setups. Because of the way the inner edge of the disc is formed, we do not have a similar criterion for the RWI at all spins, as was the case in the theoretical study in Casse \& Varniere (2018); therefore, we do not expect to have the same growth rate. Nevertheless, as we have similar initial conditions, the difference between growth rates reflects the changes in the local conditions near the last stable orbit, hence, the similarity between the lowest right plot of Figs. 1 and 3 .

If we were interested in the actual differences in the growth rate based only the spin, we would need to perform another set of simulations resolving similarly the sharpness of the edge of the disc. While this will be necessary for the study of a specific object based on specific observations, it is beyond the scope of this paper, where we are only looking at the overall impact of the RWI on the observables.

\section{Impact of the RWI upon the X-ray emission of the accretion disc}

In this section, we aim at providing observables that may be linked with the presence of the RWI within the inner part of an accretion disc at the last stable orbit of a spinning black hole. Since we are interested in the RWI as a model for HFQPOs, we first proceed to look at some of the characteristic observables used in timing analysis of black hole binaries and then at its impact upon the energy spectrum of the disc. 


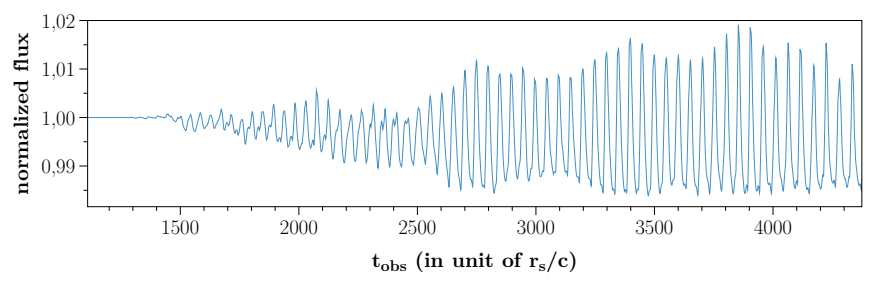

Fig. 4. X-ray lightcurve of the case $a=0.75$ computed from gyoto for an observer at infinity looking at the system under an inclination of $70^{\circ}$ (see Sect. 2 for more details about the computation model).

Using GRAMRVAC we can obtain the temperature at every point of our simulated disc and then, using gyoto, we can compute its blackbody emission and ray-trace it back to the observer. Similarly to the standard observation, we get the energy spectrum as seen by a distant observer and the overall lightcurve by integrating it over the energy range. For all the plots displayed in this study, we have set the mass of the black hole to ten solar masses while the angle of inclination of the disc is $70^{\circ}$ with respect to the observer's line of sight.

\subsection{X-ray light-curve and power density spectrum}

Among the first and most obvious observables, it is important to emphasise the ability of the RWI to modulate the X-ray flux a distant observer would receive. NOVAs was designed in order to be able to provide such observables from the numerical simulations. As an example, we present in Fig. 4 the typical X-ray light-curve obtained from a simulation where the spin parameter $a=0.75$. We can clearly see that as the amplitude of the RWI grows, the modulation of the X-ray flux amplifies.

Looking straight at the light-curve from our simulation does not provide enough information to test the possibility of the RWI as a source of HFQPOs. As we remain in the time domain, we turn our attention to the power density spectrum (PDS), which is computed as the Fourier transform of our light-curve. There is more than one reason to focus on the PDS when trying to compare our simulated observations with the real data. First of all, it is the most commonly used diagnostic for HFQPOs (see for example Varniere \& Rodriguez 2018). Secondly, the RWI has the unique characteristic that several modes can be triggered simultaneously, as can be seen in Fig. 2. This has led us, specifically in Varniere et al. (2019), to study in more detail what causes the different modes to emerge. We found that the key component for which modes becomes dominant is the local condition of the system where the instability develops. This means that the same object can exhibit different flavors of mode at different times depending on the local changes. We also found an overall relation between the maximum amplitude of the HFQPOs and the spin of the black-hole which was tentatively compared with existing observations.

While the precise method can be found in Varniere et al. (2019), here we show in Fig. 5 a few examples of the PDSs made from our simulated light curves illustrating the ability of the RWI to produce a pair of peaks in the PDS that are similar to what has been observed.

\subsection{GR effects on the disc energy spectrum of the disc}

Instead of looking at the overall light curve, we can look at the energy distribution of the flux as function of energy. One of the advantages of NOVAs is that we can turn certain aspects of the

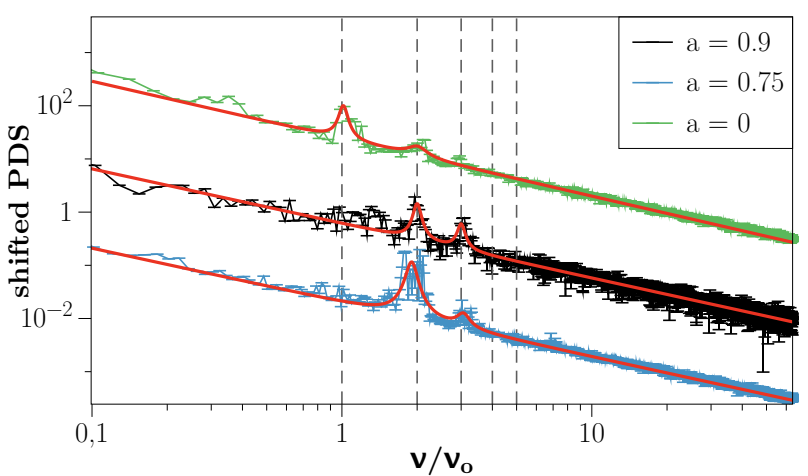

Fig. 5. PDS for three different times and three different spins showing mostly one peak, twin peaks and two peaks with very different rms. Those show some of the most commonly observed PDSs with HFQPOs.

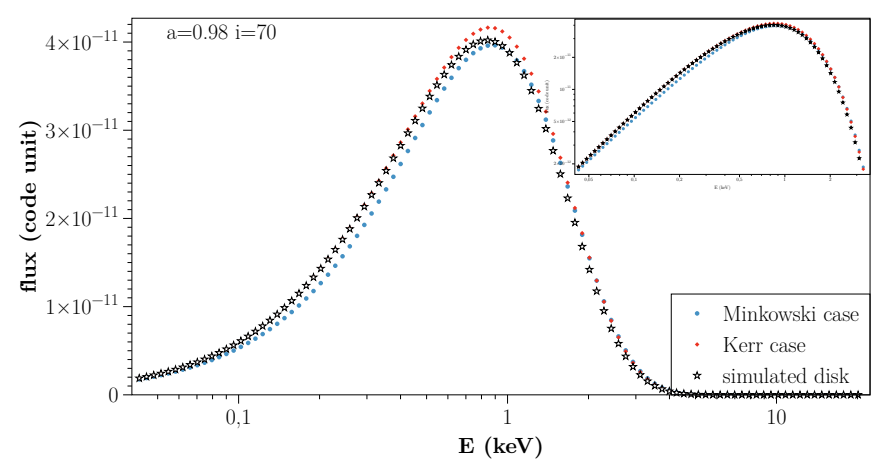

Fig. 6. Comparison between the full GR simulated spectrum (stars) of the unperturbed, pre-RWI disc with the cases of a standard disc model with either a Minkowski ray-tracing (diskbb equivalent) or a general relativistic Kerr ray-tracing for a case where $a=0.98$ with an inclination angle of $70^{\circ}$. The corner plot is the same plot in log-log.

process on and off in order to test their impact. The first test was to look at the unperturbed disc, meaning a disc without any influence exerted on it by the RWI, to see which aspect of the full GR treatment was necessary and when. To that aim, we compared the cases with full GR simulation (displayed with star symbols on Fig. 6) with the cases of a standard equivalent disc (Shakura \& Sunyaev 1973) with either a Minkowski metric (equivalent to using diskbb in xspec) or a Kerr metric for the ray-tracing.

Our Fig. 6 shows the differences between the emitted flux computed in these three cases. This figure also emphasises the fact that the full GR setup is necessary to accurately describe the emission from the accretion disc and that taking into account GR effects in the computation of the emission alone will not lead to an accurate estimate of the energy distribution spectrum of the disc.

Discarding relativistic effects could also be the reason behind the fact that it is harder to obtain good $\chi^{2}$ for the spectral fits of high-spin systems for each and every state. In order to explore the differences between high and low spins, we looked at the shape of the spectrum or, more precisely, at how the shape of the spectrum compares with the diskbb spectrum which is often used to fit spectral data. Figure 7 shows that for low spins (blue points), the full GR simulated flux is relatively close to the diskbb flux that it is fitted against, but as soon as we go to high spins, the overall shape of the spectrum starts to diverge. This would then lead to a worse result for $\chi^{2}$ for higher spins than can be achieved for lower spins. 


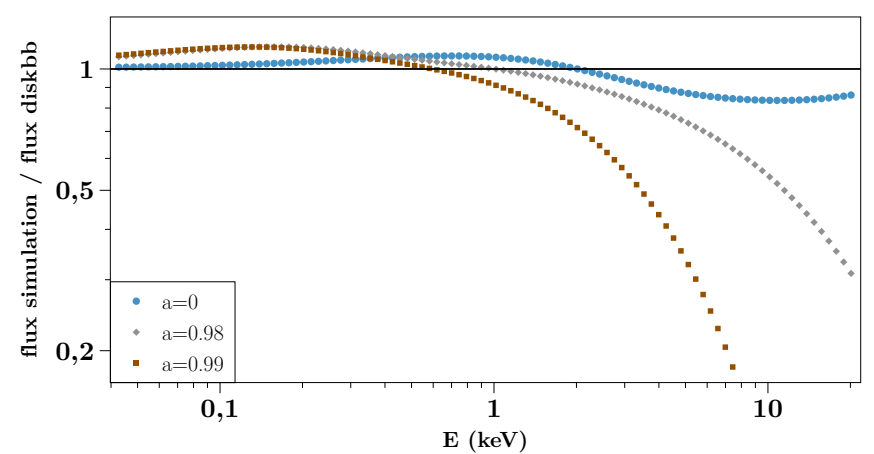

Fig. 7. Evolution as a function of energy of the simulated flux over the diskbb flux for the same system.

\subsection{Impact of the RWI on the energy spectrum}

It was surmised in Varniere et al. (2016) that QPOs could imprint the X-ray spectra of black hole binaries. Indeed, the structure at the origin of the timing modulation is hotter than the underlying disc and therefore its emission will be part of the energy spectrum. Thanks to NOVAs, we can now directly compute the energy spectrum associated with the simulations exhibiting peaks in their PDS. This will allow us to test if such an imprint exists and if it is detectable.

\subsubsection{Impact of the RWI on the emission of the disc}

We have already shown that the energy spectrum was affected by the GR effects in high spin systems, even in the absence of a structure in the disc. Here we are focusing on the impact coming from the presence of the RWI in the disc. In Fig. 2, we can see that the RWI causes a hotter non-axisymmetric structure close to the inner edge of the disc. We demonstrated, in simpler terms, in Varniere et al. (2016) that such a structure will directly impact the resulting multi-colour blackbody emission of the disc. The way such a structure impacts the total disc emission is shown on Fig. 8 where the disc spectrum is displayed without the RWI, using green circles (this is actually the disc in which the perturbations were added) in addition to one of the low points of the RWI modulation (using hollow black stars) and a high point of the RWI modulation at a late simulation time (in red stars). All the fluxes are normalised to the disc flux when no RWI is present at $0.5 \mathrm{keV}$ to simplify the comparison. We see that below $0.5 \mathrm{keV}$, the fluxes are very similar and, as the energy increases, the impact of the RWI becomes visible. Indeed, the hotter structure caused by the RWI peaks at higher energy than the surrounding disc which, in turn, causes an increasing discrepancy at higher energy. While the difference presented here seems quite pronounced, it is not straightforward to directly observe such differences because the disc emission is embedded with the emission coming from the whole system. Another difficulty here is the timescale at which those phenomena occur, meaning the timescale of the HFQPO. This is addressed in Sect. 4.4.

\subsubsection{Impact of the RWI on the comptonised energy spectrum}

As we have seen in the previous section, there is a noticeable impact coming from the RWI on the disc energy spectrum. Since we want to see if such an impact might be detected observationally, we need to compute the comptonised spectrum coming from that disc. As a first approach, we decided to use the simpl

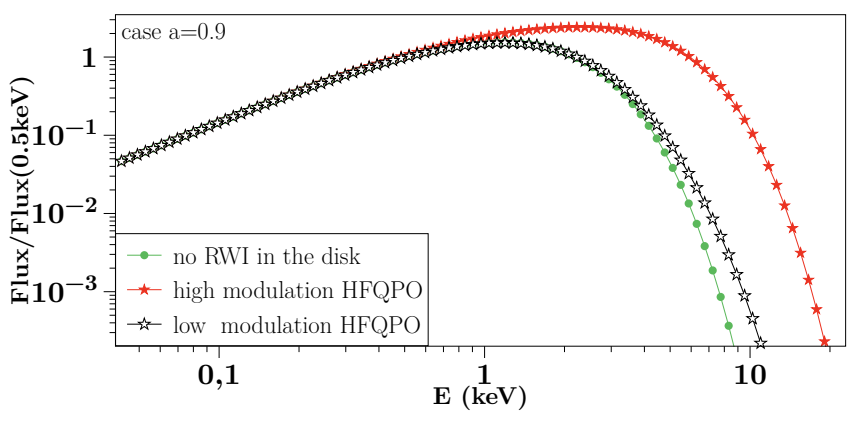

Fig. 8. Disc spectra comparison between the no RWI case (green circle) and the high (red star) and low (hollow black star) flux state of the disc when the RWI is at work in late stage of simulations. To ease the comparison, all fluxes are normalised to the no RWI flux state at $0.5 \mathrm{keV}$.

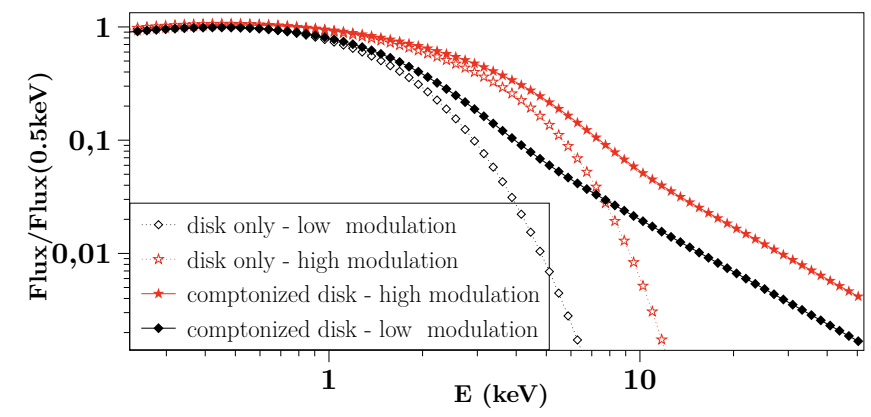

Fig. 9. Thermal and comptonised spectra of the high (red star) and low (black star) states of the RWI flux in late times of the simulation. All fluxes are normalised to their value at $0.5 \mathrm{keV}$ in order to simplify comparisons.

procedure, which is a simple comptonisation model from Steiner et al. (2009) to compute the full energy spectrum of our simulated disc. In order to choose the parameters for the corona, we selected data from the 1998-1999 outburst of XTE J1550-564 with similar disc emissions to the radiative emission computed in our simulations (Varniere \& Rodriguez 2018).

We see in Fig. 9 that adding the comptonisation process to the computation of the radiative spectrum emphasises the effect of the RWI on the energy spectrum. Indeed, the presence of the RWI in the disc shifts the maximum of the disc emission to a higher energy, which, in turn, shifts the comptonised emission, leading to a significant high-energy component beyond $5 \mathrm{keV}$. While this effect appears significant, it is not a straightforward process to be able to detect them. Indeed, in order to observe these differences, we would need to compare the energy spectra while keeping only the "top" (respectively, the bottom) part of the light curve when HFQPOs are detected, all the while accumulating enough photons to get a low signal to noise spectrum.

\subsection{Indirect detectability of the impact of the RWI}

With that difficulty in mind, we decided to look at the impact of the RWI on the shape of the energy spectrum. In order to do so, we converted our spectrum to be readable by xspec using flx $2 x s p$ while fitting our simulations with simpl(diskbb) to see how different the fit parameters are when compared to the actual values of the simulations. Here we have focussed only on the disc parameters as they are directly affected by the presence of the RWI. 

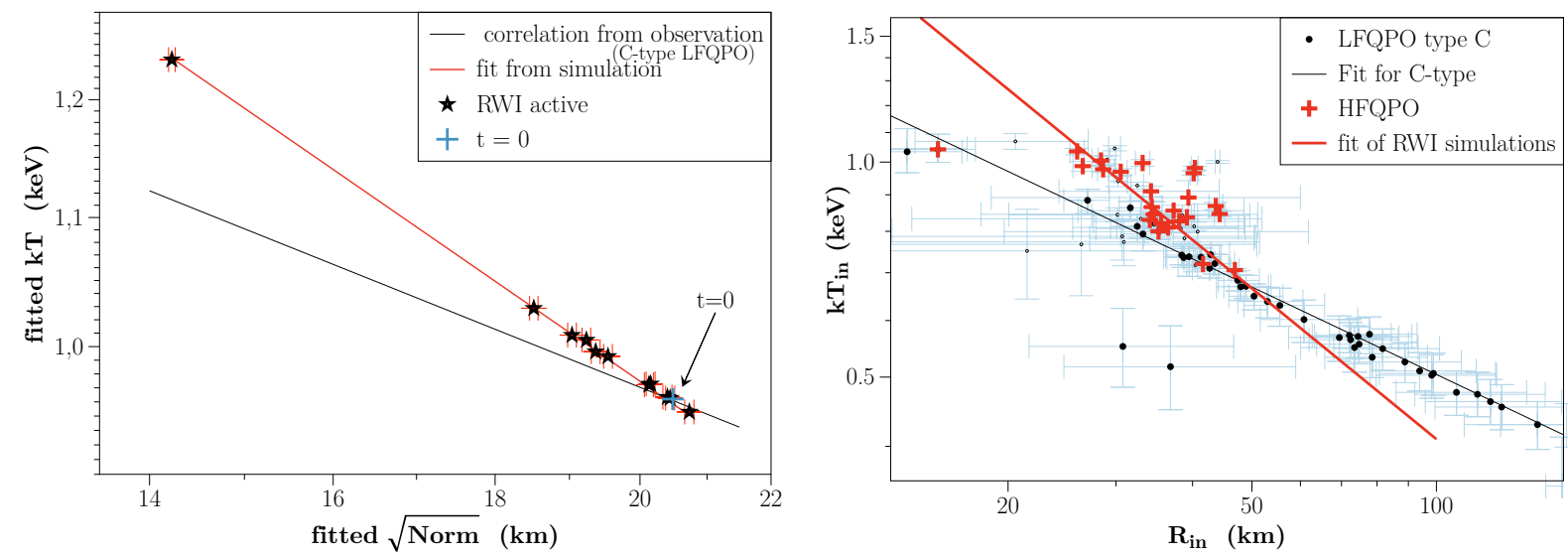

Fig. 10. $T_{\text {in }}$ versus $r_{\text {in }}$. On the left, we give the fitted value for a simulation of the RWI with $a=0.9$. The black line represents a fit of data from XTE J1550-564 in the case of the type C LFQPO. On the right, data from XTE J1550-564 with the red crosses representing the known HFQPO detections and the red line is a fit of the simulated RWI data. Observational data are from Sobczak et al. (2000) for the spectral component and Remillard et al. (2002) for the timing component.

In order to set our results in context, we use the data from XTEJ1550-564, focussing on the most common case where only type C LFQPO are observed with no HFQPOs detected ${ }^{3}$. The left of Fig. 10 shows how the fitted inner edge of the disc, with the actual inner edge remaining at the last stable orbit in the simulation, and its fitted temperature correlates when the amplitude of the RWI increases. For comparison, the black line represent a fit of data from XTEJ1550-564 in the case of a type C LFQPO with no HFQPO. Our simulations shows a clear departure from the case where type C LFQPOs, with no HFQPOs, are observed.

We went a step further and checked how that correlation obtained for a disc with an active RWI compared with observations of systems with detected HFQPOs. For that purpose, we took data from XTEJ1550-564, which has the most HFQPO detections as of this writing (Varniere \& Rodriguez 2018), and plotted $T_{\text {in }}$ versus $r_{\text {in }}$ correlations. The first correlation displayed with the black line is a fit of the type C LFQPOs with no HFQPO, while the red crosses stand for known HFQPO detections. In order to see how our RWI simulated data fare in that plot, we added the fit of our simulated RWI data coming from the left panel of Fig. 10 as a red line.

We see that the overall behavior of the fitted disc parameters originating from data with detected HFQPOs is comparable to the behavior of the fitted disc parameters of our simulations where the RWI is active at the inner edge of the disc. Such departure from the "standard" type C LFQPO correlation could be taken as an indirect observable of structure in the disc. However, such indirect detection is not enough to constrain the type of structure in the disc, nevertheless it shows the necessity to explore the impact of timing structure on the energy spectrum.

\section{Conclusion}

\subsection{Impact of the GR effects on the disc}

Using NOVAs to obtain the radiative emission from an equilibrium disc in a full GR framework, we found this emission to have an increasing departure from the diskbb prediction as the

\footnotetext{
3 We are focusing on the type C LFQPO where no HFQPOs are observed as there is a lot of data and a clear correlation.
}

spin increases. While this is not surprising as the diskbb routine does not take in account GR effects, it might be the cause for some of the high $\chi^{2}$ fits found in high spin systems. With the newer, more precise, instruments we now have, it would be interesting to study some of those previously hard to fit systems to see if taking into account more GR effects allows for better fits.

Another impact of the full GR treatment of the disc and its radiative emission is that we were able to show, for the first time, that the RWI is naturally triggered at the edge of a disc close to its last stable orbit as was predicted and previously tested in the pseudo-Newtonian context Tagger \& Varniere (2006).

\subsection{QPOs are more than just timing features}

While the main feature of the RWI lies in the peaks present in the PDS, it is interesting to explore the impact of this instability on the energy spectrum as the local emission departs from the one produced by a standard disc. Indeed, we found an impact on the disc spectrum, namely, an extended spectrum at the highest energy due to the occurence of hot RWI vortices in the disc. Moreover, this impact of the instability is propagated at higher energy through the comptonisation process. Here the difficulty lies in the fast time variability of microquasars, which makes it difficult to directly compare our predictions with observations. One way to overcome that difficulty would be to look at higher mass systems, especially tidal disruption events and other supermassive black-hole systems exhibiting QPOs. Such systems might give us access to the timing precision that we need to directly compare our simulated spectrum with observations.

In order to bypass the timescale problem entirely, we explored the possibility of any indirect detectability of those objects by looking at the impact of the changes in the spectrum on the fitted spectral parameters. Using data from XTE J1550-564, we found that a disc with an active RWI lies in the same fitted parameter space as X-ray binary systems where a HFQPO is detected. On top of that, we have also shown that there is a clear departure between RWI-active discs and systems where a type C LFQPO with no HFQPOs is detected, reinforcing the link between HFQPOs detected in the PDS and the presence of the RWI in the inner region of the disc. 
Acknowledgements. We thanks the referee for his careful reading of the manuscript. PV acknowledges financial support from the LabEx UnivEarthS (ANR-10-LABX-0023 and ANR-18-IDEX-0001). Computing was partly done using the Division Informatique de l'Observatoire (DIO) HPC facilities from Observatoire de Paris (http://dio.obspm.fr/Calcul/) and at the FACe (Francois Arago Centre) in Paris.

\section{References}

Altamirano, D., \& Belloni, T. 2012, ApJ, 747, L4

Belloni, T., Méndez, M., \& Sánchez-Fernández, C. 2001, A\&A, 372, 551

Belloni, T. M., Sanna, A., \& Méndez, M. 2012, MNRAS, 426, 1701

Casse, F., \& Varniere, P. 2018, MNRAS, 481, 2736

Casse, F., Varniere, P., \& Meliani, Z. 2017, MNRAS, 464, 3704

Kerr, R. P. 1963, Phys. Rev. Lett., 11, 237
Lovelace, R. V. E., \& Romanova, M. M. 2014, Fluid Dyn. Res., 46, 041401 Mignone, A., \& McKinney, J. C. 2007, MNRAS, 378, 1118

Remillard, R. A., \& McClintock, J. E. 2006, ARA\&A, 44, 49

Remillard, R. A., Sobczak, G. J., Muno, M. P., \& McClintock, J. E. 2002, ApJ, 564,962

Shakura, N. I., \& Sunyaev, R. A. 1973, A\&A, 24, 337

Sobczak, G. J., McClintock, J. E., Remillard, R. A., et al. 2000, ApJ, 544, 993

Steiner, J. F., Narayan, R., McClintock, J. E., \& Ebisawa, K. 2009, PASP, 121, 1279

Tagger, M., \& Varniere, P. 2006, ApJ, 652, 1457

Varniere, P., \& Rodriguez, J. 2018, ApJ, 865, 113

Varniere, P., Tagger, M., \& Rodriguez, J. 2012, A\&A, 545, A40

Varniere, P., Mignon-Risse, R., \& Rodriguez, J. 2016, A\&A, 586, L6

Varniere, P., Casse, F., \& Vincent, F. H. 2019, A\&A, 625, 8

Vincent, F. H., Meheut, H., Varniere, P., \& Paumard, T. 2013, A\&A, 551, A54 